\title{
TUBERCULOSIS DATA UPDATE
}

$\mathrm{T}$ he tuberculosis data reported in the 1991 Infectious Disease Notifications (Supplement to the June Public Health Bulletin) was an underenumeration due to late notification of cases. The Tuberculosis Register was also reviewed for missing data and updated. In addition nine records were denotified. This has resulted in a further 74 notifications for Mycobacterium tuberculosis and 35 atypical mycobacteria over those notifications reported in June 1992.

Total notifications for mycobacterial infection including tuberculosis and atypical mycobacterial infection increased from 530 in 1990 to 621 in 1991, a rise of 17 per cent. Tuberculosis notifications rose by 16 per cent for the same period, from 340 (1990) to 396 (1991). Eight reactivated cases of tuberculosis ( 2 per cent) were recorded in 1991 compared with seven reactivated cases in 1990 .

For 1991 more than 25 new cases of tuberculosis were notified for each month, except from January to March. There is evidence of continued transmission of tuberculosis in NSW. The strongest indication of this is the high level of notifications in the 0-4 age group. There is a marked male predominance in new cases aged less than five.

All Areas and Regions reported notifications for 1991. Central Sydney and South Western Sydney Area Health Services reported the highest rates of new tuberculosis, with rates of $17 / 100,000$ and $13 / 100,000$ population respectively. The overall rate for NSW was $6.8 / 100,000$. This compares with a rate of 5.7 per 100,000 for 1990 .

Tuberculosis is strongly associated with ethnicity resulting from migration patterns to NSW from countries of high tuberculosis prevalence (Table 10).

TB-HIV coinfection rates have increased from 1 per cent in 1989 to 3 per cent in 1991 (Table 11). HIV status was recorded on 377 of the 396 tuberculosis notifications received for 1991 (95 per cent). The influence of HIV infection on tuberculosis notifications in NSW has not reflected overseas experiences, where coinfection rates have been reported to be as high as 22 per cent of tuberculosis cases having coexistent HIV infection.

HIV coinfection is reported in notifications for atypical mycobacteria - 49 per cent of notifications were HIV positive in 1991 (Table 12).

\begin{tabular}{|c|c|c|c|}
\hline \multirow{2}{*}{\multicolumn{4}{|c|}{$\begin{array}{l}\text { TABLE } 10 \\
\text { TUBERCULOSIS NOTIFICATIONS BY } \\
\text { ETHNIC GROUPING, NSW, 1989-1991 }\end{array}$}} \\
\hline & & & \\
\hline Ethnic Grouping & 1989 & 1990 & 1991 \\
\hline $\begin{array}{l}\text { Aboriginal Australian } \\
\text { Non-Aboriginal }\end{array}$ & 4 & 3 & 1 \\
\hline Australian & 93 & 95 & 106 \\
\hline Asian & 140 & 151 & 184 \\
\hline European & 44 & 52 & 51 \\
\hline Pacific Islander & 13 & 14 & 15 \\
\hline Other & 17 & 25 & 20 \\
\hline Total & 311 & 340 & $377^{*}$ \\
\hline
\end{tabular}

* 19 notifications - ethnicity not known.

\begin{tabular}{|c|c|c|c|c|}
\hline \multicolumn{5}{|c|}{ TABLE 11} \\
\hline \multicolumn{5}{|c|}{$\begin{array}{l}\text { TUBERCULOSIS NOTIFICATIONS } \\
\text { BY HIV STATUS, NSW, 1989-1991 }\end{array}$} \\
\hline & 1989 & 1990 & 1991 & Total \\
\hline $\begin{array}{l}\text { HIV+ } \\
\text { HIV- }\end{array}$ & $\begin{array}{r}4 \\
307\end{array}$ & $\begin{array}{r}3 \\
337\end{array}$ & $\begin{array}{r}10 \\
367\end{array}$ & $\begin{array}{r}17 \\
1011\end{array}$ \\
\hline Total & 311 & 340 & $377^{*}$ & $1028 *$ \\
\hline
\end{tabular}

*19 notifications - HIV status not known.

TABLE 12

NOTIFICATIONS OF ATYPICAL MYCOBACTERIAL INFECTION BY HIV STATUS, NSW, 1989-1991

\begin{tabular}{|l|r|r|l|l|}
\hline & 1989 & 1990 & 1991 & Total \\
\hline HIV+ & 91 & 113 & 114 & 318 \\
HIV- & 78 & 77 & 108 & 263 \\
\hline Total & 169 & 190 & $222^{*}$ & $581 *$ \\
\hline
\end{tabular}

*3 notifications - HIV status not known.

\section{PUBLIC HEALTH EDITORIAL STAFF}

The Bulletin's editorial advisory panel is as follows:

Dr Sue Morey, Chief Health Officer, Department of Health; Professor Stephen Leeder, Professor of Community Medicine, University of Sydney; Professor Geoffrey Berry, Professor of Epidemiology \& Biostatistics, University of Sydney; Professor Ian Webster,

Professor of Community Medicine, University of NSW; Dr Christine Bennett, Associate Director, Services Planning, NSW Health

Department; Dr Michael Frommer, Epidemiologist, Epidemiology \& Health Services Evaluation Branch; Ms Jane Hall, Research Officer, Department of Community Medicine, Westmead Hospital; and Mr Michael Ward, Acting Director, Strategic Marketing, NSW Health Department.

The editor is Dr George Rubin, Director, Epidemiology and Health Services Evaluation Branch, NSW Health Department.

The Bulletin aims to provide its readers with population health data and information to motivate effective public health action. Articles, news and comments should be 1,000 words or less in length and include the key points to be made in the first paragraph.

Please submit items in hard copy and on diskette, preferably using WordPerfect 5.1.

Please send to The Editor, Public Health Bulletin, Locked Mail Bag 961, North Sydney NSW 2059, Fax (02) 3919232

Design - Health Public Affairs Unit, NSW Health Department.

Suggestions for improving the content and format of the Bulletin are most welcome. 
NEW TUBERCULOSIS NOTIFICATIONS BY MONTH, NSW, 1991

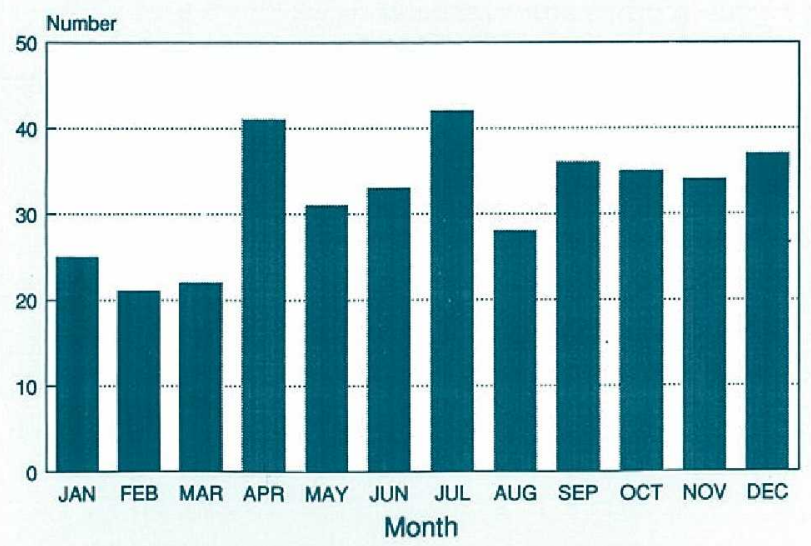

NEW TUBERCULOSIS NOTIFICATION RATE BY AHS/REGION, NSW, 1991

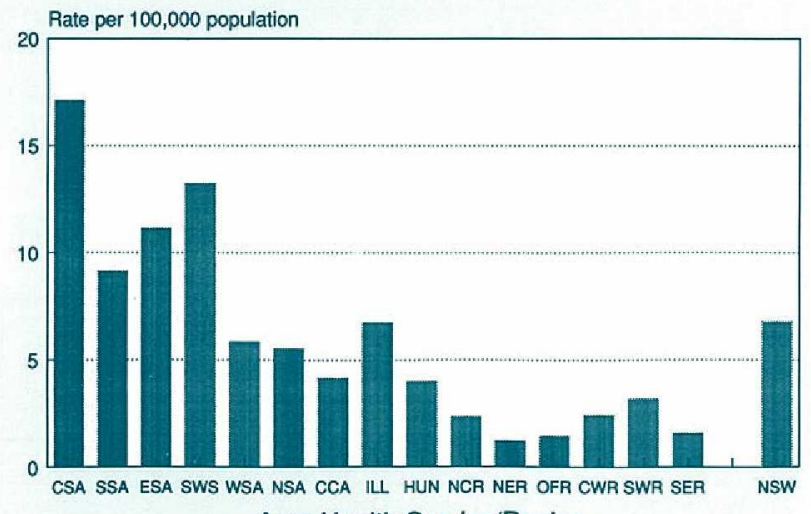
Area Health Service/Region
NEW TUBERCULOSIS NOTIFICATION RATE BY AGE AND SEX, NSW, 1991

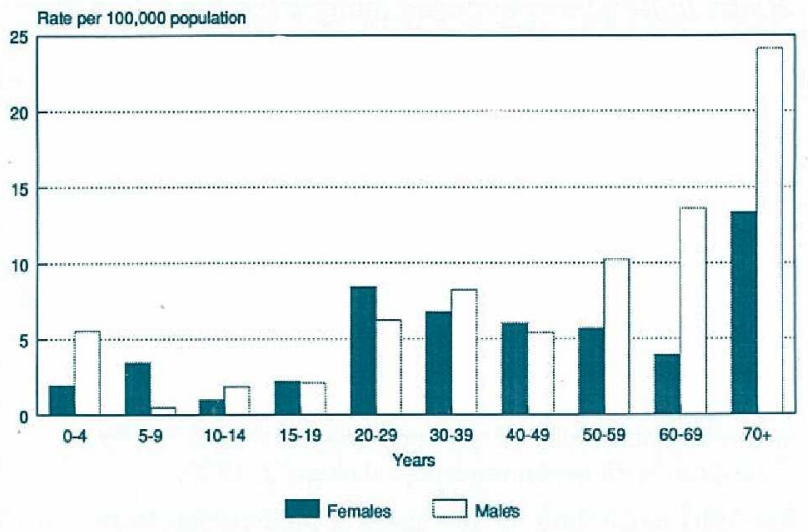

MYCOBACTERIAL INFECTION NOTIFICATIONS NSW, 1982-1991

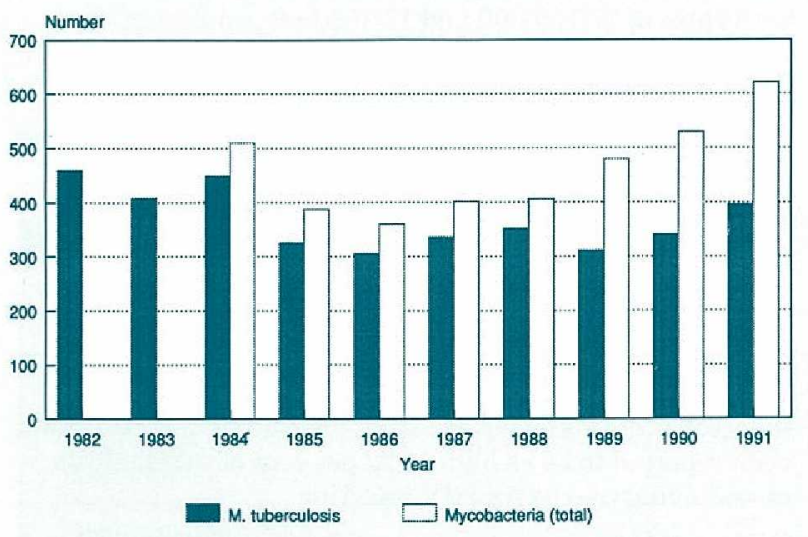

MYCOBACTERIAL AND HIV COINFECTION NSW, 1989-1991

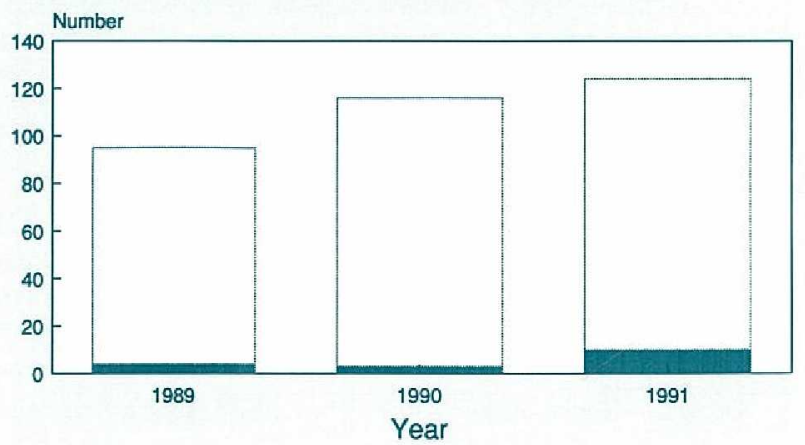

$\square$ M. tuberculosis $\square$ Atypicals 


\section{TABLE 13}

SALMONELLA NOTIFICATIONS 1991

\begin{tabular}{|c|c|c|c|c|c|c|c|c|c|c|c|c|c|}
\hline ORGANISM & Jan & Feb & Mar & Apr & May & Jun & Jul & Aug & Sep & Oct & Nov & Dec & Total \\
\hline S. aberdeen & & 1 & & & & & & & & & & & 1 \\
\hline S. adelaide & & 4 & & 1 & & 1 & 1 & & 1 & 3 & 2 & & 13 \\
\hline S. agona & & 1 & & & 1 & 3 & & 1 & & & 6 & & 12 \\
\hline S. anatum & & 3 & & 1 & 1 & & & 1 & 4 & 1 & & & 11 \\
\hline S. arizonae & & & & & & & & & 1 & 2 & 1 & & 4 \\
\hline S. berta & 2 & & & & & & & & & 1 & 1 & & 4 \\
\hline S. birkenhead & & & & & & & & & & & & 1 & 1 \\
\hline S. blockley & & & & 1 & & & & & 1 & & & & 2 \\
\hline S. bovis morbificans & 6 & 31 & 4 & 4 & 13 & 6 & 8 & 2 & 3 & 1 & 7 & 4 & 89 \\
\hline S. braenderup & 1 & & & & & & & & & & & & 1 \\
\hline S. brandenburg & 2 & & & & & & & & & & & & 2 \\
\hline S. bredeney & & & & & & 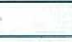 & 1 & & & & & & 1 \\
\hline S. cerro & & & & & 2 & 6 & 4 & 1 & 4 & 8 & 1 & & 26 \\
\hline S. charity & & & & & & & & & 1 & & & & 1 \\
\hline S. chester & 1 & 2 & 1 & & 1 & & & 1 & 1 & & & 2 & 9 \\
\hline S. cubana & & 1 & & & & & & & & & & & 1 \\
\hline S. derby & & 1 & & & & & 1 & 3 & & 2 & 1 & 3 & 11 \\
\hline S. eastbourne & 1 & & & & & & & & 1 & & & & 2 \\
\hline S. emek & 1 & & & & & 1 & & & & & & 1 & 3 \\
\hline S. enteritidis & & 1 & 1 & & 3 & 1 & & & 1 & & 1 & 1 & 9 \\
\hline S. eppendorf & & & & & & & & & & & & 1 & 1 \\
\hline S. give & 1 & & & & 1 & 2 & & 2 & & & & & 6 \\
\hline S. hadar & 1 & & & & 1 & & & 2 & & 2 & 1 & & 7 \\
\hline S. hamburg & & 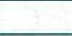 & & & & & & & 1 & 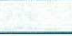 & & & 1 \\
\hline S. havana & 2 & 1 & & & 1 & & & & 1 & 2 & 1 & & 8 \\
\hline S. heidelberg & 5 & 11 & & 4 & 2 & 2 & 1 & 1 & 4 & 1 & 2 & 4 & 37 \\
\hline S. hindmarsh & & 1 & & & & & & & & & & & 1 \\
\hline S. ibadan & & & & & & 1 & & & & & & & 1 \\
\hline S. idikan & & 1 & & & & & & & & & & & 1 \\
\hline S. infantis & 1 & 4 & & 1 & 2 & 2 & 1 & 2 & 1 & 2 & 1 & 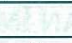 & 15 \\
\hline S. java bioser & 1 & 2 & & & & & & 1 & 1 & & 1 & 1 & 7 \\
\hline S. kottbus & & 3 & 2 & & & 1 & 1 & & 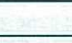 & 1 & & 4 & 8 \\
\hline S. krefeld & & & & & & 1 & & & & & & & 1 \\
\hline 5. lagos & 1 & & & & & & & & & & & & 1 \\
\hline S. lexington & & & & & & 1 & & & & & & (e & 1 \\
\hline S. mbandaka & & & & & & 1 & & 1 & & & & & 2 \\
\hline S. meleagridis & & & & & & & & & 1 & & 1 & & 2 \\
\hline S. montevideo & 1 & & & & & & & 1 & & 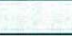 & & & 2 \\
\hline 5. morehead & & & & & & & & & & & & 1 & 1 \\
\hline S. muenchen & 1 & 1 & 2 & & 1 & & & 1 & & & & 2 & 8 \\
\hline S. newport & & & & & & 1 & & 1 & & & 1 & & 3 \\
\hline S. ohlstedt & & & & & & & & & 1 & & & & 1 \\
\hline S. oranienburg & & & & & & & & & & & & 1 & 1 \\
\hline S. orion & & & 2 & & & & & & & & 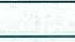 & 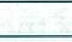 & 2 \\
\hline S. paratyphi A & & 2 & & & & & & & & & 1 & & 3 \\
\hline S. rissen & & & & & 1 & & & & & & & & 1 \\
\hline S. saint paul & & 2 & 1 & & 1 & & 1 & 1 & 1 & 3 & & 3 & 13 \\
\hline S. schwerzeng & 2 & & 1 & & & & & & & & & & 3 \\
\hline S. senftenberg & 1 & & & & 1 & & & & & 1 & & & 3 \\
\hline S. singapore & 2 & 1 & 1 & 2 & & 2 & & & 1 & & & & 9 \\
\hline S. species untyped & & & 2 & 7 & 2 & 2 & 9 & 2 & 3 & 8 & 7 & 8 & 50 \\
\hline S. sofia & & & & & & & & & & & 1 & & 1 \\
\hline S. stanley & & & & & & & & & 1 & - & 1 & 2 & 4 \\
\hline S. tennessee & 1 & 1 & 1 & 1 & & & & & & 1 & & 1 & 6 \\
\hline S. thompson & & & & & & & 1 & & & & & & 1 \\
\hline S. typhi & & & & & & & & & & & 4 & & 4 \\
\hline S. typhimurium & 25 & 28 & 7 & 10 & 18 & 22 & 23 & 19 & 18 & 28 & 41 & 21 & 260 \\
\hline S. virehow & & & & & & & & & & & & 1 & 1 \\
\hline S. wandsworth & 1 & & & & & & & 1 & & 1 & & & 3 \\
\hline S. wangata & & & & & & & & 1 & 1 & & & & 2 \\
\hline S. waycross & 2 & 1 & & & & & & 1 & & & 1 & & 5 \\
\hline S. welikade & & & & & & & & & 1 & & 1 & & 2 \\
\hline S. weltevreden & & & & & & & & & & & 1 & & 1 \\
\hline Total & 62 & 104 & 26 & 32 & 52 & 56 & 52 & 46 & 54 & 66 & 86 & 58 & 693 \\
\hline
\end{tabular}

